\title{
A Comparative Analysis of MMPI and Rorschach Findings Assessing Combat-Related PTSD in Vietnam Veterans
}

\author{
—Analysis of MMPI and Rorschach Findings Assessing PTSD
}

\author{
Ioanna Katsounari ${ }^{1}$, Jordan Jacobowitz ${ }^{2}$ \\ ${ }^{1}$ Social Work Department, Frederick University, Nicosia, Cyprus; \\ ${ }^{2}$ The Chicago School of Professional Psychology, Chicago, USA. \\ Email: jkatsounari@yahoo.com, soc.ki@fit.ac.cy, jjacobowitz@thechicagoschool.edu \\ Received March $17^{\text {th }}$, 2011; revised April 20 ${ }^{\text {th }}, 2011$; accepted May 22 $2^{\text {nd }}, 2011$.
}

\begin{abstract}
There has been a proliferation of assessment research on Post-traumatic Stress Disorder (PTSD) over the past twenty years. In spite of recent advances in the PTSD assessment research, there continues to be a controversy as to whether the MMPI or Rorschach is more useful in determining the presence of PTSD. The present comparative analysis of the research literature will carefully evaluate controlled empirical studies, which utilized psychometric measures such as the MMPI/2 and Rorschach to identify PTSD in Vietnam Veterans. This analysis is guided by the paucity of comparative data for standardized objective and projective instruments to assess combat-related PTSD. The analysis indicated that the MMPI as an assessment instrument focuses on symptom recognition of PTSD while the Rorschach seems to be more likely to identify chronic adaptations to trauma. The significance of pre-combat factors, such as preexisting personality, and their impact on the way individuals make meaning and express traumatic experiences needs to be further addressed in future research. The need for reliable and valid measures to assess combat-related PTSD is urgent as an increasing number of soldiers return from war zones.
\end{abstract}

Keywords: PTSD, Combat Trauma, PTSD Assessment, Rorschach, MMPI

\section{Introduction}

The heterogeneity and complexity of symptoms that can be indicated in PTSD is often difficult to distinguish from other psychiatric diagnoses, many of which are characterized by the same symptoms. Indeed, some researchers have seriously questioned the legitimacy of PTSD as a psychiatric diagnosis except for "pure" cases when only disorder-specific symptoms are found and no Axis II diagnoses are observed (Hyer et al., 1986).

The need for psychological measures that fully capture the dynamics and vicissitudes of traumatic stress responses to combat is urgent. An increasing number of returning soldiers from war torn areas need to be evaluated for the diagnosis of PTSD. Very relevant to the current article is the fact that there is frequently a "delayed reaction" between initial and later screenings of returning soldiers in the proportions of those reporting mental health problems. For soldiers in the reserves, for instance, the percentage of soldiers reporting mental health problems nearly doubled (from $12.7 \%$ to $24.5 \%$ ) between the time of returning from Iraq to three to six months later (DeAngelis, 2008). Psychological instruments that can reliably identify psychiatric problems as soon as possible would contribute to earlier and possibly more efficacious interventions.

In the early and late 70's, procedures for the assessment of PTSD consisted mainly of clinical interviews that evaluated the presence of or absence of a DSM (Diagnostic and Statistical Manual of Mental Disorders) diagnosis. In addition, questionnaire and structured interviews were employed to assess pre- military, military, and post-military history (Keane \& Fairbank, 1983). It was not until the early 80 's that some studies began to investigate the use of objective psychological tests, such as the Minnesota Multiphasic Personality Inventory (MMPI/MMPI-2) to aid in the diagnosis of PTSD. Less work has been done using projective testing, and specifically the Rorschach, to help diagnose PTSD patients. The Rorschach is a promising tool in that it can detect varying levels of war-related stress in soldiers and address the complex symptomatology inherent in traumatic states often not readily accessible on self-report measures (Sloan, Arsenault, \& Hilsenroth, 2002).

This comparative analysis of assessment measures is especially valuable since it scrutinizes whether the most popular psychometric techniques assess the interactive effects of premorbid personality variables, personal resource variables, and the nature of the stressor dimensions in a particular traumatic event. The particular population (Vietnam Veterans) was selected because of the plethora of studies conducted using these two instruments to assess both acute but also chronic trauma. Psychlit searches were conducted using terms such as Vietnam Veterans and PTSD, MMPI/2 and PTSD, Rorschach and PTSD. The content and methodology of the studies relevant to the analysis were then reviewed.

\section{MMPI and PTSD}

Several studies have attempted to identify a combat-related PTSD profile on the MMPI. Most studies show a high-point code of 8-2 with a moderately elevated F-scale score to be sug- 
gestive of combat-related PTSD diagnosis (Fairbank, Keane, \& Malloy, 1983; Wilson \& Walker, 1990; Albrecht et al., 1994). The F, 2-8/8-2 code is representative of a combat-related PTSD sample for the MMPI-2 as it has been for the MMPI. However, Albrecht et al., (1994) pointed out that it is possible for some differences to be seen on the Harris-Lingoes subscale scores due to their increased variability, and this increased variability must be noted by clinicians who are comparing scores from the MMPI-2 with the MMPI.

In addition, the MMPI validity scales (L, F, and $\mathrm{K}$ ) are higher for PTSD patients as opposed to control groups. Researchers have cautioned against generalizing this mean profile configuration at the individual patient level. They suggested that individual profile configurations show considerable variability for highpoint and two point codetype (Albrecht et al., 1994; Baldrachi et al., 1999; Fairbank, Keane, \& Malloy, 1983; Munley, Bains, Bloem, \& Busby, 1995; Wilson \& Walker, 1990).

Scale 8 elevations among male Vietnam veterans diagnosed with PTSD reflect to a large extent difficulties with depression and dissociation. However, a significant proportion of variance in scale 8 scores is also accounted by both non-overt and overt psychotic symptoms. Clinicians should be cautious not to misconstrue MMPI-2 scale 8 scores as strong signs of psychosis among PTSD sufferers. However, psychotic symptoms should be carefully assessed in PTSD veterans as well. It has also been suggested that when the symptoms of a severe stress disorder like PTSD are present, depressive symptomatology may occur as a direct function of the inability of individuals to engage in reinforcing activities (Burke \& Mayer, 1984; Elhai et al., 2003).

The psychasthenia scale (7) has also been found to be elevated among Vietnam Veterans, indicating the tendency to ruminate about the trauma and experience episodes of traumatic imagery. The elevated three-point code (2-8-7) suggests obsessive rumination about trauma-related imagery and affect in persons suffering from PTSD. In addition, MMPI clinical scales $(2,8,7,6)$ appear to be assessing the DSM-III-R symptom clusters of intrusion, avoidance, and physiological hyperarousal (Wilson \& Walker, 1990).

In terms of the content scales, PTSD sufferers score significantly higher on the Anger (ANG) scale and on the Social Discomfort Scale (SOD). The high scores found on the SOD and ANG scales appear consistent with the social alienation and anger control problems often seen clinically in combat veterans with PTSD (Glenn et al., 2002).

The elevations studies have found on the validity, clinical, and subscales of the MMPI seem to identify a global PTSD profile that overlaps with other affective and anxiety disorders. Research has raised important questions in terms of what PTSD comorbidity means given the current classification system. It is highly likely that most PTSD combat veterans present with a complex diagnostic picture. A very important consideration would be whether different diagnostic groups are distinct groups or overlapping manifestations of the same group (e.g. PTSD + Anxiety vs PTSD + Depression). Most commonly, these groups receive treatment based on the assumption that they share more behavioral similarities than differences. Researchers argued that this inadequacy resides in the categorical nature of the DSM-III-R, which assumes that human behavior patterns can be divided into clearly delineated groups with dis- crete and non-overlapping types of behavior. However, most of PTSD symptoms overlap with at least one another diagnostic category within the DSM-III-R. MMPI research can prove very useful in distinguishing between the various comorbid groups and develop distinct therapeutic interventions based on their unique presentations (Weyermann, Norris, \& Hyer, 1996).

The PTSD (Pk) scale was found to be significantly higher on the MMPI-2 than on the MMPI. The PTSD scale (PK) has been found to be high in both face validity and content validity for the PTSD symptom complex, and its use improves on the diagnostic hit rates obtained from the validity and clinical scales alone. At present, this scale is considered to represent the best effort at PTSD measurement among Vietnam veterans (Keane, Malloy, \& Fairbank, 1984). However, research has at times shown conflicting findings when different studies attempted to cross-validate this scale. The best conclusion is one of caution where the use of MMPI-PTSD scale is concerned. In psychiatric populations, the PTSD MMPI measures may be useful in ruling out the diagnosis of PTSD, but high scores may suggest only further attempts to be made to establish the diagnosis (Hyer et al., 1987; Munley et al., 1995). In addition, PTSD optimal cutting scores might vary with the co-existence of substance abuse, the presence of specific referral for PTSD treatment and race (Watson, Kucala, \& Manifold, 1986).

In addition, the PTSD (Ps) scale has been found to be more robust in the differentiation of PTS symptomatology in a group of outpatient Vietnam combat veterans (Baldrachi et al., 1999). Overall, utilizing the MMPI-2, and specifically the PS and PK scales, could aid in the initial assessment of such individuals, thereby contributing to a multimodal evaluation to assess for the presence or severity of PTSD symptom (Watson et al., 1986; Gayton et al., 1986; Cannon et al., 1987).

Findings also suggested that symptom overreporting is often part of veterans suffering from PTSD. However, although studies showed that these veterans respond to both the obvious and neutral items at a rate higher than did the other groups, they do not respond differentially between obvious and neutral items. One can therefore argue that PTSD veterans are not differentially endorsing or overreporting symptoms and that regardless of compensation, one need not imply negative features with symptom overreporting (Tolin et al., 2004).

The data also suggested that the Fp scale is less sensitive to psychopathology than are alternate overreporting indices such as the F, Fb, F-K, Ds, and O-S, and therefore, may be of greater utility in the assessment of PTSD in veterans. Therefore, Fp may be a more valid measure of overrepoting as it is less likely to be artificially elevated for individuals in extreme distress because of frank psychopathology (Tolin et al., 2004).

To conclude, it appears that current research practices may not yield definitive estimates of symptom overreporting among veterans evaluated for PTSD. This is because severely impaired patients are more likely to seek compensation. In addition, CS veterans not diagnosed with PTSD may suffer from psychiatric disorders other than PTSD, therefore it would be inappropriate to conclude that these individuals are faking PTSD. The important issue is how this symptom feature is part of PTSD for Vietnam Veterans. Researchers suggested that once acceptance of the disorder is legitimized in inpatient settings, PTSD veterans feel free to express their problems. By doing so, veterans can reaffirm their identity, band with other veterans, and make 
sense out of confusing symptoms (Hyer, Fallon, Harrison, \& Boudewyns, 1987). Therefore, a multi-team, multi-modal, multi-assessment approach to the diagnosis of PTSD in combat veterans is necessary to ensure that correct identification of malingering veterans is made (Tolin et al., 2004).

\section{Rorschach and PTSD}

Projective methods, such as the Rorschach, offer some distinct contributions for assessing combat-related PTSD. They are often less direct and intrusive than objective tests, which helps circumvent the guardedness of trauma survivors. Re-experiencing the traumatic events through recollections, nightmares, or flashbacks can severely interfere with the cognitive processes set in motion by the Rorschach, and particularly with the capacity to perceive events objectively and to think logically (Ephraim, 2002). Studies using the Exner scoring system have found the Rorschach useful in the assessment of civilians with PTSD and in identifying PTSD in nonveteran groups of adults (Sloan, Arsenault, \& Hilsenroth, 2002). However, to this date only a limited number of published studies used the Rorschach in the assessment of PTSD focusing on U.S. combat veterans of the Vietnam War. Findings in regards to the assessment of PTSD in Vietnam Veterans using the Rorschach have shown contradictory results.

Earlier research supported that guilt over combat atrocities appears to be the basic trauma as indicated in the content analysis of the Rorschach (Salley \& Teiling, 1984). Later research indicated that Rorschach protocols show an abreactive repetition of the trauma, with the inkblots serving only as the stimuli in the process. These findings have suggested that PTSD sufferers when presented with ambiguous or affectively charged stimuli they react to current situations as a recurrence of the traumatic stress. Specifically, chromatic cards (II, III, VIII, IX, $\mathrm{X})$ seem to provoke uncontrolled and apparently trauma- related experiences (Van der Kolk \& Ducey, 1989).

In terms of the veteran's experience type, the research has shown inconsistent findings. Part of the research has indicated that Vietnam veterans are classified as extratensive (Van der Kolk \& Ducey, 1989; Souffront, 1987; Swanson et al., 1990). Extratensive protocols of Vietnam veterans have been found to be characterized by extensive and gory blood and anatomy content, uncensored and uncontrolled references to traumatic Vietnam experiences, high number of inanimate movement (m) responses, and an absence of integrated whole $\left(\mathrm{Q}^{++}\right.$and $\left.\mathrm{W}+\right)$ location and developmental quality responses (Van der Kolk \& Ducey, 1989).

Coartative protocols of Vietnam veterans show very few responses, no use of color, and few or no $\mathrm{M}$ responses. These were interpreted as evidence of the veterans' inability to integrate immediately affective experience and to structure experience through higher cognitive processes. The extratensive and coartated Rorschach records suggest the failure of active ego adaptation, one in the direction of overwhelmed undercontrol, the other in the direction of rigid overcontrol. These traumatized men lacked the internal processing mechanisms that might lead to the integration of trauma (Van der Kolk \& Ducey, 1989).

Other studies have shown that a large group of Vietnam veterans falls into the introversive and ambitensive category of experience type (Goldfinger et al., 1998; Hartman et al., 1990). These findings suggested that Vietnam veterans suffering from PTSD use a less efficient problem solving and coping style (ambitent EB) than controls, are less likely to use a more efficient coping style (introversive EB), and have more mental access to combat-related imagery than non-PTSD Vietnam veterans, although few appear preoccupied with gory, traumatic scenes. These researchers argued that this could suggest that in the course of chronic PTSD, preoccupation with traumatic imagery may attenuate over time, with periodic reemergences during episodes of exacerbated symptomatology.

Overall, it has been found that PTSD sufferers show a higher proportion of color to movement scores. It has been suggested that the PTSD sufferers experience affective stimulation in excess of their capacity to process, control, and delay the impact of trauma through the "higher" symbolic capacities for thinking, reflection, planning, and perspective-taking (Souffront, 1987; Van der Kolk \& Ducey, 1989).

One further finding is that PTSD subjects show an extraordinarily high number of inanimate movement responses (Van der Kolk \& Ducey, 1989). The results indicated that the inanimate movement response was the best discriminator between veterans with PTSD and veterans without PTSD (Souffront, 1987). This again indicated that PTSD patients experience a significant amount of tension, discomfort, and situational stress. Another notable finding in this research was the veterans' very low tolerance for stress (Hartman et al., 1990; Swanson et al., 1990).

In terms of the accuracy and specificity of form quality, the PTSD sufferers showed an interesting combination of (1) heavy emphasis on conventional ("ordinary") form at the expense of sharp and accurate perception, and (2) a very high proportion of the amorphous (formless) categories. This combination appears to be a counterpart of the duality of response to trauma, representing the biphasic cognitive processing of traumatic experience. Rorschach's of the Vietnam Veterans confirmed the clinical impression that people with severe PTSD are incapable of modulated affective experience; they either respond to affective stimuli with intensity, which is appropriate only to the traumatic situation, or they barely react at all (Van der Kolk \& Ducey, 1989).

Another finding was the tendency of these patients to view reality in an unconventional manner and often distort reality in their perception of situations. Notable was also their inclination toward an oversimplified view of situations. Under stress, these patients were likely to distort reality, however, this distortion was not psychotic. They were able to perceive reality in a conventional manner (Swanson et al., 1990).

Vietnam veterans were also likely to display affect in an unmodulated manner, which amplified their impulsivity. As a result, they tended to avoid emotionally laden situations. Although they seemed uninterested in people, and often were perceived by others as cold and distant, they were not particularly lonely. Isolating themselves from others may help them minimize their exposure to emotionally laden situations, thereby decreasing the possibility of finding themselves in situations where they may behave impulsively (Swanson et al., 1990).

Overall, studies to this date researching the association of PTSD to specific Rorschach scores have shown discrepancies, 
which will only be resolved through further control studies larger in scale and more diverse in demographics and traumatic history. These methodological improvements will allow a constellation of Rorschach variables to emerge consistently across studies as indicators of cognitive and emotional sequelae of traumatic reactions to combat exposure. The variables that have been found consistently on these studies include intense and poorly modulated affect, impulsivity, unconventional reality testing, and inconsistent problem solving.

\section{Conclusions and Future Research Suggestions}

Research indicates that the Rorschach does not consistently or globally measures self-reported characteristics as does the MMPI (Meyer, 1996). Subsequently, it appears that we should not be comparing these two measures. PTSD on the MMPI is really PTSD as it is consciously reported by the patient. On the other hand, PTSD on the Rorschach is PTSD as it is manifest in implicit perceptual propensities and qualities of verbal articulation (Meyer, 1997).

Rorschach scores do not typically measure constructs that reside within conscious awareness. It has been suggested that to the extent that Rorschach constructs are not viewed as consistently tapping conscious and deliberately reported phenomena, clinical interpretations will be more accurate (Meyer, 1996).

Individuals who have experienced prolonged and repeated trauma display massive efforts to protect their psyche, which involve defense mechanisms such as denial, dissociation, avoidance, and repression (Herman, 1997). The Rorschach seems to be more likely to identify such chronic adaptations to trauma.

Brende (1983) suggested that the pronounced identity changes observed in Vietnam veterans bear a notable similarity to the disorders of self typically found in borderline and narcissistic patients. Hartman et al., (1990) has indicated in his research that for his group of Vietnam veterans, the mean Rorschach Schizophrenic Index was in the range reported for borderline and schizotypal populations. Again, the Rorschach seems to provide access to covert personality traits that may not surface on the MMPI. Additional research is needed to explore the relationship between combat-related PTSD and borderline personality characteristics.

Shatan (1973) identified guilt feelings and self-punishment as the first theme of most concern to Vietnam veterans. In the research reviewed, guilt arising from participating in or witnessing inhumane actions in combat was only noted in one Rorschach study. Objective tests, such as the MMPI, seem to leave out the personal subjective quality of the veteran's experience. The second theme of feeling of being scapegoated is not noted in any of the tests, but it might be related to scale 6 (paranoia) elevations in the MMPI.

This analysis has suggested that considering the possibility of coexistence of PTSD and other, more traditional, forms of psychopathology exacerbated or triggered by combat experience is important. Both MMPI and Rorschach have indicated that there is a high degree of comorbidity with PTSD, particularly with depression and anxiety. The presence of comorbid disorders often complicates the diagnostic picture of PTSD on the assessment tool. Evaluators of combat related PTSD need to consider the possibility of co-existing symptomatology that may not fit the diagnostic criteria for PTSD.

When the results of the Rorschach and the MMPI are somewhat inconsistent or contradictory, the clinician must make the decision to emphasize certain aspects of the test findings, while suppressing results from other sources of test data. In determining which results to emphasize, we must consider the relative reliability and validity of the specific data sources. Finn's (1996) suggestions for combining MMPI and Rorschach results are highly recommended when such discrepancies are found.

Contradictory findings between the two tests underscores the importance of reviewing other data sources, such as interview data and psychosocial history data in reaching clinical conclusions. Using a multi-method approach in the multidimensional assessment of PTSD symptomatology should be the ideal approach.

The findings of the assessment literature suggest the possibility of unique PTSD subtypes within Vietnam veterans. Therefore, each veteran may display a different symptom picture. Subsequently, it is important to emphasize the individual as the unit of analysis.

The development of PTSD is integrally related to how the individual experienced specific combat events. The significance of pre-combat factors in determining how individuals make meaning of combat experience is not mentioned in the literature. Preexisting personality needs to be assessed as an integral part of the meaning the veteran will give to his combat experience, and directly affect the form and expression of his posttraumatic stress disorder. This does not imply that a personality disorder was present in the veteran or would have developed it without exposure to combat. It is more indicative of the importance of close examination of pre-combat, combat, and post-combat factors in attempting to understand the meaning of war experience to the individual.

War trauma has been directly conceptualized in the literature as combat exposure. Most studies define war trauma and combat exposure as comprising a single dimension. The definition of combat and war trauma has been based on a traditional understanding of conflict in which certain territorial areas are held by hostile troops and the proximity to those areas reflects the level of combat exposure of the veteran. Some veterans had been asked to place themselves in categories such as "moderate," "heavy," "low" combat experience without attempting to establish the objective comparability of these evaluations across individuals (Laufer et al., 1984). This traditional definition of war trauma needs to be re-conceptualized to include other forms of combat situations that can potentially be traumatizing.

Locus of control (external and internal) is another variable addressed in some of the studies reviewed. This is different from helplessness in that it relates to the confidence in one's ability to affect their problems (Hyer et al., 1987). It appears that investigating this variable may yield important data as to the veteran's coping style and vulnerability to depression and is a critical issue to be addressed in therapy.

The use of psychometric inventories presupposes a working knowledge of the psychological and social issues pertinent to the veteran from a specific war zone. A working relationship with the evaluator is critical for the open discussion of traumatic events, atrocities, and guilt-inducing memories. The use of a structured interview that encompasses pre-combat military personality factors, a military history, and post-combat adjust- 
ment is critical when assessing PTSD.

In addition, knowing what combat has meant to the veteran is critical both in understanding the way in which the disorder is manifested and in working with the veteran psychotherapeutically to resolve the impact that traumatic combat experiences have on his post-combat life.

The current managed care environment demands empirical data to develop evidence-based outcomes. Rorschach and MMPI data can greatly facilitate the clinicians' ability to better understand the individual's cognitive, emotional, and interpersonal resources. Although, projective measures, like the Rorschach, have been at times deemed inappropriate for providing that type of data, this review of the studies has shown otherwise. The Rorschach's ability to assess an individual's psychological resources indicates that it can provide a unique window through which to observe subtle psychological variations. The combined outcome data from both instruments can help contribute to imperatives for good clinical standards of care and fiscally responsible services for veterans with combat-related PTSD.

The studies reviewed presented with several methodological and theoretical limitations. First and foremost, most of the studies have used small sample sizes. Administering, scoring, and interpreting the Rorschach could be an arduous task for researchers aspiring to conduct large scale studies. However, until more studies with larger sample sizes are conducted, it is inappropriate to generalize the findings of smaller studies.

It is important for the evaluator to take into consideration whether the veterans are assessed in an inpatient as opposed to an outpatient setting. This may play a significant role because it may provide essential information as to the severity of the PTSD, the presence of other psychological disorders, the services the veteran is currently receiving, and the willingness of the veteran to admit his emotional struggles. It becomes increasingly challenging for the researcher to discern whether the Rorschach findings are a reflection of the PTSD syndrome or a reflection of a comorbid condition instead. Although it is likely that outpatient veterans may also suffer from a comorbid condition, the decreased severity of this condition may affect the Rorschach findings to a lesser degree.

In addition, the profiles of veterans assessed by the Keane et al. (1984) scale will differ depending on whether the assessment was conducted in an inpatient vs. an outpatient setting. This is because the Keane et al scale was normed on an outpatient population and the cut off score represents those standards.

Participants in several of the studies were taking some type of psychotropic medication (antidepressants, anxiolytics, neuroleptics). In addition, many had secondary diagnoses. Often, the symptomatology they presented with was part of a comorbid condition rather than a clear cut PTSD disorder. The findings do not help us discern whether the assessment findings are rooted in PTSD, simply reflect these veterans being chronic PTSD patients, or whether these patients have charactereological problems, which flavor their PTSD.

Most of the studies reviewed in this analysis did not specify the type of combat that the veteran was exposed to. Combat status as defined in most of the studies did not necessarily reflect frequency and intensity of combat experience. There are different degrees of combat exposure and they need to be assessed carefully as they may determine the degree of PTSD. In addition, studies have not looked into the differences in the diagnostic picture of Vietnam veterans suffering from delayed versus chronic versus acute trauma. It is highly likely that the different diagnostic groups may reveal distinct MMPI profiles.

MMPI profile configurations do not take into account individual variations. Some individual variations addressed in the research are race and socioeconomic status. Other variations to be taken into account include gender, ethnicity, education, religion, and age.

All the studies reviewed have failed to take into consideration precombat personality influences. Crucial premorbid personality influences include the veteran's personality structure before exposure to combat, preexisting psychopathology, personal worldview, locus of control, life experiences, and personal attributions.

Researchers need to conduct more studies assessing Rorschach variables with acute symptoms of PTSD. Psychological assessment soon after a war allows for potentially greater recall of events, and the more extreme emotions associated with these experiences. In addition, research has shown that data collected within six months of exposure to trauma reduced the likelihood that the individual may minimize, distort, or exaggerate experiences or symptoms (Sloan et al., 2002). The Rorschach is currently widely used in VA hospitals with newly returning soldiers from war zones.

Differences in combat exposure need to be carefully assessed as they may have an impact on the severity of PTSD symptoms shown on the Rorschach. The amount of graphic details (morbid, anatomy, violence, aggression, explosion, blood etc) on the Rorschach is likely to increase with more direct and intensive combat exposure.

The studies showed discrepancies in terms of the coping style (extratensive, introversive, ambitent) reported. These differences likely stem from participant selection as well as methodological differences between the various studies. These variables need to be closely monitored in future studies.

Among the Rorschach studies reviewed, the researchers failed to consider precombat personality differences that may have impacted the findings. Although, some researchers noted the possible interference of demographic variables with the Rorschach results, the influence of precombat personality is not mentioned. As with the MMPI studies reviewed, this is a common gap in the literature that has not been addressed to this date.

It is important to interpret Rorschach findings in the context of current PTSD theory, especially pertaining to combat-related situations. The traumatized veterans' anxiety, depression, and somatic symptoms are not the same as ordinary anxiety, depression, and somatic disorders. In addition, the cognitive impairment indicated on the Rorschach is not ordinary impairment. It is crucial that the Rorschach is scored and interpreted as trauma-related. The criticisms of the studies also seem to underscore the need for changes in some current Rorschach scoring and interpretation guidelines when it comes to cases of severe combat related PTSD. For example, the veteran's cognitive disturbances associated to intrusive symptoms and the trauma-related nature of their symptoms should be directly acknowledged by the scoring system and interpreted accordingly. Current interpretive strategies fail to adequately assess the veteran's struggle by reducing symptomatology to a personality style or an ego-syntonic character trait (Ephraim, 2002). 
A major issue is the difficulty of differentiating between manifestations of severe PTSD as opposed to psychosis, and schizophrenia in particular. Future studies can be conducted using these two instruments where individuals with severe PTSD are compared to a schizophrenia-diagnosed patient comparison group.

Somatic concerns can be part of the PTSD syndrome and veterans may endorse more health complaints. There is a need for further research on the effectiveness of projective measures in assessing the complex relationship among physical and psychological symptoms in individuals with war-related stress. Future research that uses stricter diagnostic coding of groups may prove valuable in illuminating the complexities of PTSD and in enhancing our knowledge regarding its treatment.

The development of PTSD is integrally related as to how the individual experienced the specific combat events. The significance of precombat factors in determining how individuals make meaning of combat experience is not mentioned in the literature. Preexisting personality needs to be assessed as an integral part of the meaning the veteran will give to his combat experience and directly affect the form and expression of his posttraumatic stress disorder.

A working relationship with the evaluator is crucial for the open discussion of traumatic events, atrocities, and guilt-inducing memories. The use of a structured interview that encompasses precombat military personality factors, a military history, and postcombat adjustment is critical when assessing PTSD. In addition, the traditional definition of war trauma needs to be reconceptualized to include other forms of combat situations that can potentially be traumatizing.

Rorschach and MMPI data can greatly facilitate the clinicians' ability to better understand the individual's cognitive, emotional, and interpersonal resources. Although, projective measures, like the Rorschach, have been at times deemed inappropriate for providing that type of data, this analysis of the studies has shown otherwise. The Rorschach's ability to assess an individual's psychological resources indicates that it can provide a unique window through which to observe subtle psychological variations. The combined outcome data from both instruments can help contribute to imperatives for good clinical standards of care and fiscally responsible services for veterans with combat-related PTSD.

The question yet remains as to whether it is appropriate to be comparing the two measures. PTSD on the MMPI is PTSD as it is consciously reported by the individual. On the other hand, PTSD on the Rorschach is PTSD as it manifests through implicit and unconscious propensities of verbal articulation. Can one argue then that they constitute measures of the same construct, but simply represent conscious vs. unconscious articulations of it? Or do they measure a completely different construct better defined as a spectrum of disorders, which is yet to be clearly captured by traditional diagnostic systems? The continuing understanding of the basic processes which underlie the development of PTSD will likely resolve the uncertainties that punctuate the study of trauma.

\section{References}

Albrecht, N. N., Talbert, F. S., Boudewyns, P. A, Touze, J., Albrecht, J. W., Hyer, L. A., \& Lemmon, C. R. (1994). A comparison of MMPI and MMPI-2 in PTSD assessment. Journal of Clinical Psychology, $50,578-585$.

doi:10.1002/1097-4679(199407)50:4<578::AID-JCLP2270500413> 3.0.CO; $2-\mathrm{X}$

Baldrachi, R., Hilsenroth, M., Arsenault, L., Sloan, P., \& Walter, C. (1999). MMPI-2 assessment of varying levels of posttraumatic stress in Vietnam combat veterans. Journal of Psychopathology and Behavioral Assessment, 21, 109-116. doi:10.1023/A:1022104405161

Burke, H. R., \& Mayer, S. (1984). The MMPI and the post-traumatic stress syndrome in Vietnam era veterans. Journal of Clinical Psychology, 41, 152-156.

doi:10.1002/1097-4679(198503)41:2<152::AID-JCLP2270410204> 3.0.CO;2-3

Cannon, D. S., Bell, W. E., Andrews, R. H., \& Filkenstein, A. S. (1987). Correspondence between MMPI posttraumatic measures and clinical diagnosis. Journal of Personality Assessment, 51, 517-521. doi:10.1207/s15327752jpa5104_3

DeAngelis, T. (2008). PTSD Treatments grow in evidence, effectiveness. Monitor, 39, 40.

Ephraim, D. (2002). Rorschach trauma assessment of survivors of torture and state violence. Rorschachiana, 25, 58-76.

Elhai, J. D., Frueh, B. C, Gold, P. B., Hammer, M. B., \& Gold, S. N. (2003). Posttraumatic stress, depression and dissociation as predictors of MMPI-2 scale 8 scores in combat veterans with PTSD. Journal of Trauma and Dissociation, 4, 51-64. doi:10.1300/J229v04n01 04

Fairbank, J. A., Keane, T. M., \& Malloy, P. F. (1983). Some preliminary data on the psychological characteristics of Vietnam veterans with Posttraumatic Stress Disorders. Journal of Consulting and Clinical Psychology, 51, 912-919. doi:10.1037/0022-006X.51.6.912

Gayton, W. F., Burchstead, G. N., \& Matthews, G. R. (1986). An investigation of the utility of an MMPI Posttraumatic Stress Disorder subscale. Journal of Clinical Psychology, 42, 916-918. doi:10.1002/1097-4679(198611)42:6<916::AID-JCLP2270420616> 3.0.CO;2-6

Glenn, M. D., Beckham, J. C., Sampson, W. S., Feldman, M. E., Hertzberg, M. A., \&Moore, S. D. (2002). MMPI-2 profiles of Gulf and Vietnam veterans with chronic Posttraumatic Stress Disorder. Journal of Clinical Psychology, 58, 371-381. doi:10.1002/jclp.1149

Goldfinger, D. A., Amdur, R. L., \& Liberzon, I. (1998). Rorschach patterns of response in Vietnam veterans with Posttraumatic Stress Disorder versus combat and normal controls. Depression and Anxiety, 8, 104-111. doi:10.1002/(SICI)1520-6394(1998)8:3<104::AID-DA2>3.0.CO;2-Y

Hartman, W. L., Clark, M. E., Morgan, M. K., Dunn, V. K., Fine, A. D., Perry, G. G., \& Winsch, D. L. (1990). Rorschach structure of a hospitalized sample of Vietnam veterans with PTSD. Journal of Personality Assessment, 54, 149-159. doi:10.1207/s15327752jpa5401\&2_15

Herman, J. (1997). Trauma and recovery. New York: Basic Books.

Hyer, L., Boudewyns, P. A., \& O’Leary, W. C. (1987). Key determinants of the MMPI- PTSD subscale: Treatment considerations. Journal of Clinical Psychology, 43, 337-340. doi:10.1002/1097-4679(198705)43:3<337::AID-JCLP2270430306> 3.0.CO;2-U

Hyer, L., Fallon, J. H., Harrison, W. R., \& Boudewyns, P. A. (1987). MMPI overreporting by Vietnam combat veterans. Journal of Clinical Psychology, 43, 79-83.

doi:10.1002/1097-4679(198701)43:1<79::AID-JCLP2270430110>3. $\underline{0 . \mathrm{CO} ; 2-\mathrm{P}}$

Hyer, L., O’ Leary, W. C., Saucer, R. T., Blount, J., Harrison, W. R., \& Boudewyns, P. A. (1986). Inpatient diagnosis of Posttraumatic Stress Disorder. Journal of Consulting and Clinical Psychology, 54, 698-702. doi:10.1037/0022-006X.54.5.698

Keane, T. M., \& Fairbank, T. K. (1983). Survey analysis of combat-related stress disorders in Vietnam veterans. American Journal of Psychiatry, 140, 348-350.

Keane, T. M., Malloy, P. F., \& Fairbank (1984). Empirical development of an MMPI subscale for the assessment of combat-related 
Posttraumatic Stress Disorder. Journal of Consulting and Clinical Psychology, 52, 888-891. doi:10.1037/0022-006X.52.5.888

Meyer, G. J. (1996). The Rorschach and MMPI: Toward a more scientifically differentiated understanding of cross-method assessment, Journal of Personality Assessment, 67, 558-578. doi:10.1207/s15327752jpa6703_11

Munley, P. H., Bains, D. S., Bloem, W. D., \& Busby, R. M. (1995). Posttraumatic stress and the MMPI-2. Journal of Traumatic Stress, 8 , 171-179. doi:10.1002/jts.2490080113

Salley, R. D., \& Teiling, P. A. (1984). Dissociated rage attacks in a Vietnam veteran: A Rorschach study. Journal of Personality Assessment, 48, 98-104. doi:10.1207/s15327752jpa4801_17

Shatan, C. F. (1973). The grief of soldiers: Vietnam combat veterans' self-help movement. American Journal of Orthopsychiatry, 43, 460-652.

Sloan, P., Arsenault, L., \& Hilsenroth, M. (2002). Use of the Rorschach in the assessment of war-related stress in military personnel. Rorschachiana, 25, 86-122.

Souffront, E. M. (1987). The use of the Rorschach in the assessment of Posttraumatic Stress Disorder among Vietnam combat veterans. Doctoral Dissertation, Philadelphia, Pennsylvania: Temple University.

Swanson, G. S, Blount, J., \& Bruno, R. (1990). Comprehensive system
Rorschach data on Vietnam veterans. Journal of Personality Assessment, 54, 160-169. doi:10.1207/s15327752jpa5401\&2_16

Tolin, D. F., Maltby, N., Weathers, F. W., Litz, B. T., Knight, J., \& Keane, T. M. (2004). The use of the MMPI-2 Infrequency-Psychopathology scale in the assessment of Posttraumatic Stress Disorder in male veterans. Journal of Psychopathology and Behavioral Assessment, 26, 23-29. doi:10.1023/B:JOBA.0000007453.24885.39

Van der Kolk, B. A., \& Ducey, C. P. (1989). The psychological processing of traumatic experience: Rorschach patterns in PTSD. Journal of Traumatic Stress, 2, 259-275. doi:10.1002/jts.2490020303

Watson, C. G., Kucala, T., \& Manifold, V. (1986). A cross validation of the Keane and Penk MMPI scales as measures of Posttraumatic Stress Disorder. Journal of Clinical Psychology, 42, 727-732. doi:10.1002/1097-4679(198609)42:5<727::AID-JCLP2270420508> 3.0.CO;2-4

Weyermann, A. G., Norris, F. H., \& Hyer, L. A (1996). Examining comorbidity and Posttraumatic Stress Disorder in a Vietnam veteran population using the MMPI-2. Journal of Traumatic Stress, 9, 353-360. doi:10.1002/jts.2490090216

Wilson, J. P., \& Walker, A. J. (1990). Toward an MMPI trauma profile. Journal of Traumatic Stress, 3, 151-168. doi:10.1002/jts.2490030111 